\title{
A Non-Perturbative Chiral Approach for Meson-Meson Interactions *
}

\author{
J.A. Oller, E. Oset ${ }^{\mathrm{i}}{ }^{\dagger}$ F. Guerrero ${ }^{\text {b }}$ J.R. Peláez ${ }^{c}$ \\ aDepartamento de Física Teórica \\ Universidad de Valencia, 46100 Burjassot (Valencia), Spain \\ ${ }^{\mathrm{b}}$ Centro Universitario Estema \\ Parque Tecnológico, 46980 Valencia, Spain \\ ${ }^{\mathrm{c}}$ Departamento de Física Teórica \\ Universidad Complutense, 28040 Madrid, Spain
}

A non-perturbative method [1] which combines constraints from chiral symmetry breaking and coupled channel unitarity is used to describe meson-meson interactions up to $\sqrt{s} \lesssim 1.2 \mathrm{GeV}$, extending in this way the range of applicability of the information contained in Chiral Perturbation Theory $(\chi P T)$ [2], since this perturbative series is typically restricted to $\sqrt{s} \lesssim 500 \mathrm{MeV}$. The approach uses the $\mathcal{O}\left(p^{2}\right)$ and $\mathcal{O}\left(p^{4}\right) \chi P T$ Lagrangians. The seven free parameters resulting from the $\mathcal{O}\left(p^{4}\right)$ Lagrangian are fitted to the experimental data. The approach makes use of the expansion of $T^{-1}$ instead of the amplitude itself as done in $\chi P T$. The former expansion is suggested by analogy with the effective range approximation in Quantum Mechanics and it appears to be very useful. The results, in fact, are in good agreement with a vast amount of experimental analyses [3, 4].

The amplitudes develop poles corresponding to the $f_{0}(980), a_{0}(980), \rho(770), K^{*}(890)$, the octet contribution to the $\phi, f_{0}(400-1200) \equiv \sigma$ and $\kappa$ 《. The total and partial decay widths of the resonances are also well reproduced.

\section{Introduction}

$\chi P T$ is the low energy effective theory of the strong interactions. It is given as a power expansion of the external four-momenta of the pseudo-Goldstone bosons $\pi, K$ and $\eta$ on the scale $\Lambda_{\chi P T} \approx 1 \mathrm{GeV}$. As a result, the expansion is typically valid up to $\sqrt{s} \lesssim 500 \mathrm{MeV}$. However, the constraints coming from the spontaneous/explicit chiral symmetry are not restricted to the low energy region [5]. In this work, we present a way of resummation of the $\chi P T$ series that in fact can be applied to any other system whose dynamics can be described by low energy chiral Lagrangians. We describe the successfull application of such approach to meson-meson interactions which are well reproduced up to $\sqrt{s} \lesssim 1.2$ $\mathrm{GeV}$.

*Talk given at PANIC99, Uppsala (Sweden), June 10-16, 1999.

${ }^{\dagger}$ Work partially supported by DGICYT under contract PB96-0753 and by the EEC-TMR ProgramContrac No. ERBFMRX-CT98-0169. J.A.O. acknowledges financial support from the Generalitat Valenciana. 


\section{Formalism}

Let us a consider a partial wave amplitude $T$ with definite isospin $(I)$. We use a matrix formalism in order to deal with coupled channels. In this way $T$ will be a matrix whose element $i j$ represents the scattering of $i \rightarrow j$ with angular momentum $L$ and isospin $I$. If we consider only two body intermediate states unitarity with coupled channels reads in our normalization:

$\operatorname{Im} T^{-1}=\rho$

where $\rho$ is a diagonal matrix with elements $\rho_{i}=\frac{p_{i}}{8 \pi \sqrt{s}} \theta\left(s-\left(m_{1 i}+m_{2 i}\right)^{2}\right.$ with $p_{i}$ the center mass three-momentum, $m_{1 i}$ and $m_{2 i}$ are the masses of the particles in the state $i$ and $\theta(x)$ is the usual Heaviside function. Eq. (11) is a well known result and is the basis of the $K$ matrix formalism since all the dynamics is embodied in $\operatorname{Re} T^{-1}$ which is $K^{-1}$. The former equation shows clearly that, when considering $T^{-1}$, unitarity is exactly satisfied with two body intermediate states.

From the $\chi P T$ expansion of $T=T_{2}+T_{4}+\mathcal{O}\left(p^{6}\right)$, where $T_{2}$ and $T_{4}$ are the $\mathcal{O}\left(p^{2}\right)$ and $\mathcal{O}\left(p^{4}\right)$ contributions respectively, we work out the expansion of $T^{-1}$. In this way we will obtain our approach for the $K$ matrix (or $\operatorname{Re} T^{-1}$ ).

$$
\begin{aligned}
T^{-1} & =\left[T_{2}+T_{4}+\ldots\right]^{-1}=T_{2}^{-1} \cdot\left[1+T_{4} \cdot T_{2}^{-1}+\ldots\right]^{-1} \\
& =T_{2}^{-1} \cdot\left[1-T_{4} \cdot T_{2}^{-1}+\ldots\right]=T_{2}^{-1} \cdot\left[T_{2}-T_{4}\right] \cdot T_{2}^{-1}
\end{aligned}
$$

Inverting the former result, one obtains:

$$
\begin{aligned}
T & =T_{2} \cdot\left[T_{2}-T_{4}\right]^{-1} \cdot T_{2} \\
K & =T_{2} \cdot\left[T_{2}-\operatorname{Re} T_{4}\right]^{-1} \cdot T_{2}
\end{aligned}
$$

\section{3. $\pi \pi$ and $K \bar{K}$ coupled amplitudes}

In [3] we study the $(I, L)=(0,0),(1,1)$ and $(2,0)$ partial waves. To make use of eq. (3) one needs the lowest and next to leading order $\chi P T$ amplitudes. In our case the $\pi \pi \rightarrow \pi \pi$ and $\pi \pi \rightarrow K \bar{K}$ are taken from [6] and the $K \bar{K} \rightarrow K \bar{K}$ is also given in [3]. Our amplitudes depend on six parameters $L_{1}, L_{2}, L_{3}, L_{4}, L_{5}$ and $2 L_{6}+L_{8}$ which are fitted to the elastic $\pi \pi(I, L)=(0,0)$ and $(1,1)$ phase shifts.

In the following table we show the resulting values for the $L_{i}$ coefficients comparing them with the $\chi P T$ values.

With the former values for the $L_{i}$ couplings we also calculate other scattering parameters in good agreement with experiment.

It is worth to indicating that from eq. (3) the $\chi P T$ expansion is recovered for low energies up to $\mathcal{O}\left(p^{4}\right)$. In this way, we also calculate in [3] the scattering lengths with values in agreement with $\chi P T$ and experiment. 


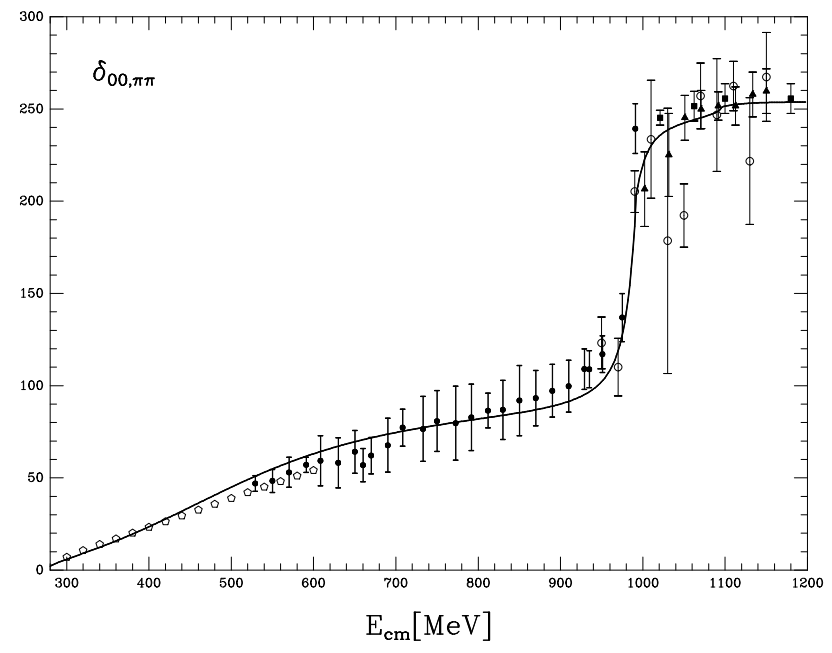

Figure 1. Elastic P-wave $\pi \pi$ phase shifts. References in [3].

Table 1

$L_{i}$ coefficients.

\begin{tabular}{ccc}
\hline & Fit & $\chi P T$ \\
\hline$L_{1} 10^{3}$ & $0.72_{-0.02}^{+0.03}$ & $0.4 \pm 0.3$ \\
$L_{2} 10^{3}$ & $1.36_{-0.05}^{+0.02}$ & $1.4 \pm 0.3$ \\
$L_{3} 10^{3}$ & $-3.24 \pm 0.04$ & $3.5 \pm 1.1$ \\
$L_{4} 10^{3}$ & $0.20 \pm 0.10$ & $-0.3 \pm 0.5$ \\
$L_{5} 10^{3}$ & $0.0_{-0.4}^{+0.8}$ & $1.4 \pm 0.5$ \\
$\left(2 L_{6}+L_{8}\right) 10^{3}$ & $0.00_{-0.20}^{+0.26}$ & $0.5 \pm 0.7$ \\
\hline
\end{tabular}

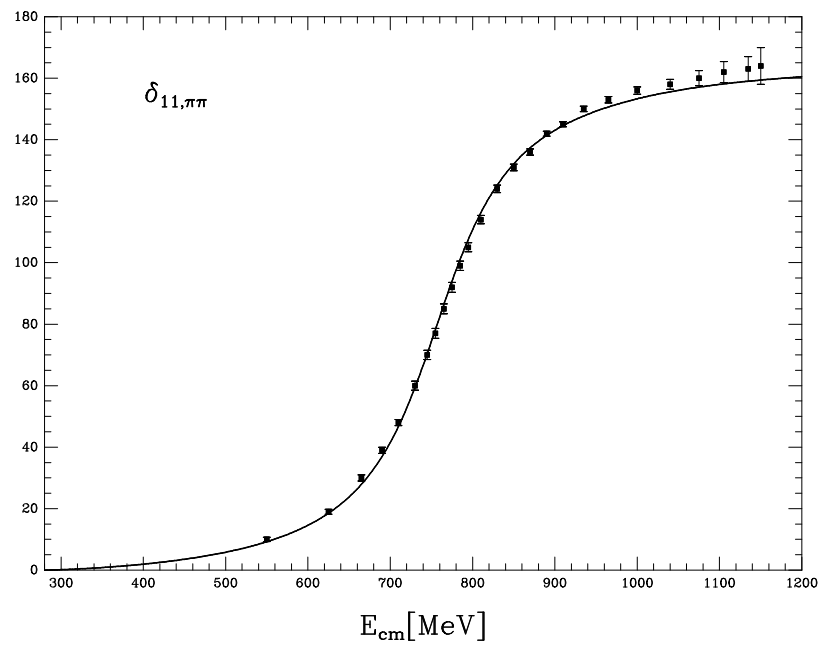

Figure 2. Elastic S-wave $I=0 \pi \pi$ phase shifts. References in [3].

\section{S and P-wave meson-meson scattering amplitudes}

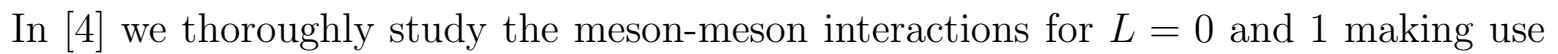
of eq. (3). However, in this case there are a lot of channels whose $\chi P T T_{4}$ amplitudes have not been calculated yet \$. The calculation of the $T_{4}$ although straightforward is cumbersome. As a result, we approximate the $T_{4}$ amplitude as in [4]

$T_{4} \approx T_{4}^{P}+T_{2} \cdot g(s) \cdot T_{2}$

where $T_{4}^{P}$ is the polynomial part of the amplitude which is essential for the vector channels (this is another way to see Vector Meson Dominance [7]) and $T_{2} \cdot g(s) \cdot T_{2}$ takes into account unitarity in coupled channels, mostly important for the scalar channels. The $g(s)$ function is a diagonal matrix whose elements are the loop integral with two meson propagators:

$g_{i}(s)=i \int \frac{d^{4} q}{(2 \pi)^{4}} \frac{1}{q^{2}-m_{1 n}^{2}+i \epsilon} \frac{1}{(P-q)^{2}-m_{2 n}^{2}+i \epsilon}$

\footnotetext{
${ }^{3}$ Even more, when this work was done the $K \bar{K} \rightarrow K \bar{K}$ amplitudes were not calculated.
} 
We regularize it making use of a cut-off $q_{\max }$.

With respect to a full $\chi P T$ calculation we are neglecting in eq. (4) the tadpole contribution (which numerically usually results to be small [6]) and the unphysical cuts (which correspond to singularities away from the physical region and hence they give rise to soft contributions which we reabsorb in the $L_{i}$ couplings).

After inserting eq. (4) with eq. (5) in eq. (3) we obtain the final expression for the $T$ matrix as in [4]. We reproduce in that work a vast amount of experimental data (phase shifts and inelasticites) for the $\mathrm{S}$ and $\mathrm{P}$-wave meson-meson scattering. We also study the mass, widths and partial decay widths of the resonances (poles) present in our amplitudes:

Table 2

Masses and partial widths in $\mathrm{MeV}$.

\begin{tabular}{|c|c|c|c|c|c|c|}
\hline $\begin{array}{c}\text { Channel } \\
(I, J)\end{array}$ & Resonance & $\begin{array}{c}\text { Mass } \\
\text { from pole }\end{array}$ & $\begin{array}{c}\text { Width } \\
\text { from pole }\end{array}$ & $\begin{array}{c}\text { Mass } \\
\text { effective }\end{array}$ & $\begin{array}{c}\text { Width } \\
\text { effective }\end{array}$ & $\begin{array}{c}\text { Partial } \\
\text { Widths }\end{array}$ \\
\hline$(0,0)$ & $\sigma$ & 442 & 454 & $\approx 600$ & very large & $\pi \pi-100 \%$ \\
\hline$(0,0)$ & $f_{0}(980)$ & 994 & 28 & $\approx 980$ & $\approx 30$ & $\begin{array}{c}\pi \pi-65 \% \\
K \bar{K}-35 \%\end{array}$ \\
\hline$(0,1)$ & $\phi(1020)$ & 980 & 0 & 980 & 0 & \\
\hline$(1 / 2,0)$ & $\kappa$ & 770 & 500 & $\approx 850$ & very large & $K \pi-100 \%$ \\
\hline$(1 / 2,1)$ & $K^{*}(890)$ & 892 & 42 & 895 & 42 & $K \pi-100 \%$ \\
\hline$(1,0)$ & $a_{0}(980)$ & 1055 & 42 & 980 & 40 & $\begin{array}{c}\pi \eta-50 \% \\
K \bar{K}-50 \%\end{array}$ \\
\hline$(1,1)$ & $\rho(770)$ & 759 & 141 & 771 & 147 & $\pi \pi-100 \%$ \\
\hline
\end{tabular}

\section{Conclusion}

We have presented a method of resummation of the $\chi P T$ series based in the expansion of $T^{-1}$. In this way unitarity is fulfilled to all orders and resonances are well reproduced. The method is rather general and could be applied to any system whose dynamics is described by chiral Lagrangians. We have applied it successfully to describe the $\mathrm{S}$ and P-wave meson-meson amplitudes giving rise to the resonances: $f_{0}(980), a_{0}(980), \rho(770)$, $K^{*}(890)$, the octet contribution to the $\phi, f_{0}(400-1200) \equiv \sigma$ and $\kappa$.

\section{REFERENCES}

1. J. A. Oller, E. Oset and J. R. Peláez, Phys. Rev. Lett. 16 (1998) 3452.

2. J. Gasser and H. Leutwyler, Nucl. Phys. B 250 (1985) 465, 517, 539.

3. F. Guerrero and J. A. Oller, Nucl. Phys. B 537 (1999) 459.

4. J. A. Oller, E. Oset and J. R. Peláez, Phys. Rev. D 59 (1999) 074001.

5. J. V. Steele, H. Yamagishi and I. Zahed, Nucl. Phys. A 615 (1997) 305; M. R. Pennington and J. Portoles, Phys. Lett. B 344 (1995) 399.

6. V. Bernard, N. Kaiser and U. G. Meissner, Nucl. Phys. B 357 (1991) 129.

7. G. Ecker, J. Gasser, A. Pich and E. de Rafael, Nucl. Phys. B 321 (1989) 311. 